BMJ Open

Diabetes

Research

\& Care

\title{
Risk of gastrointestinal bleeding and cardiovascular events due to NSAIDs in the diabetic elderly population
}

\author{
Jungmee Kim, ${ }^{1}$ Joongyub Lee, ${ }^{2}$ Cheol Min Shin, ${ }^{3}$ Dong Ho Lee, ${ }^{3}$ Byung-Joo Park ${ }^{1}$
}

To cite: Kim J, Lee J, Shin CM, et al. Risk of gastrointestinal bleeding and cardiovascular events due to NSAIDs in the diabetic elderly population. BMJ Open Diabetes Research and Care 2015;3:e000133. doi:10.1136/bmjdrc-2015000133

Received 13 July 2015 Revised 28 October 2015 Accepted 1 November 2015

\section{(1) CrossMark}

${ }^{1}$ Department of Preventive Medicine, Seoul National University College of Medicine, Seoul, Republic of Korea

${ }^{2}$ Medical Research

Collaborating Center, Seoul National University Hospital and Seoul National University College of Medicine, Seoul, Republic of Korea

${ }^{3}$ Department of Internal Medicine, Seoul National University Bundang Hospital, Seongnam, Republic of Korea

Correspondence to Dr Dong Ho Lee; dhljohn@gmail.com

\section{ABSTRACT}

Objective: We assessed gastrointestinal bleeding (GIB) and cardiovascular (CV) risks such as myocardial infarction or stroke associated with non-steroidal antiinflammatory drug (NSAID) use among elderly patients with diabetes.

Methods: Using a nationwide claims database covering 2008-2012, we conducted a cohort study of patients with diabetes aged $\geq 65$ years. Among the 117610 patients, NSAID users and non-users were propensity score matched, excluding any who had experienced a potentially confounding event in the year prior to cohort entry. Multivariate Cox regression models treating death as competing risk were used.

Results: There were $2184(1.86 \%)$ cases of GIB and NSAID users had an adjusted HR (aHR) of 1.68 (95\% CI 1.54 to 1.83) of GIB risk after adjusting for age, sex, comorbidities and recent medications compared to NSAID non-users. There were 9333 (7.94\%) cases of myocardial infarction or stroke with an aHR of 1.20 (95\% Cl 1.15 to 1.25$)$. The risk of GIB was higher in patients with liver disease and renal failure, while that of CV events was higher in patients who received anticoagulants, antiplatelet agents, aspirin and selective serotonin reuptake inhibitors. The number needed to harm was 111 for GIB and 77 for CV events. Among different NSAIDs, nimesulide increased the risk of GIB and ketorolac increased the risk of $\mathrm{CV}$ events compared to celecoxib (aHR 2.60 and 3.13, respectively).

Conclusions: Elderly patients with diabetes treating NSAIDs had a significantly higher risk of both upper GIB and CV events compared to NSAID non-users, and the risk varied among different NSAIDs regardless of cyclooxygenase-2 activity.

\section{INTRODUCTION}

While non-steroidal anti-inflammatory drugs (NSAIDs) are the most widely used drugs, especially among the elderly, who frequently use them on a long-term basis, the benefitrisk balance of individual NSAIDs is chiefly driven by their gastrointestinal and cardiovascular (CV) safety profile, and there have been many studies regarding each outcome. In a recent meta-analysis, the pooled adjusted rate ratio of gastrointestinal complications ranged from 1.81 to 4.22 , while the

\section{Key messages}

- In elderly patients with diabetes, non-steroidal anti-inflammatory drug (NSAID) use was associated with a higher risk of both upper gastrointestinal bleeding and cardiovascular events such as myocardial infarction or stroke.

- Gastrointestinal bleeding risk was higher in patients with liver disease or renal failure, and cardiovascular event risk was higher in patients with a recent history of receiving aspirin or selective serotonin reuptake inhibitors.

- NSAID complications varied among different NSAIDs regardless of cyclooxygenase-2 activity.

rate ratio for CV complications was estimated around 1.3 among NSAIDs including celecoxib. ${ }^{1}$ However, diabetes is a well-known risk factor for CV outcomes which has also been suggested to be an independent risk factor for peptic ulcer bleeding, contributing a population attributable fraction of $4 \%{ }^{2}$ Moreover, the 2014 Standards of Medical Care in Diabetes recommend aspirin as a primary prevention for those with increased $\mathrm{CV}$ event risk. ${ }^{3}$ However, according to the American College of Gastroenterology Guidelines for Prevention of NSAID-Related Ulcer Complications (ACG Guideline), concurrent use of any one drug among aspirin, corticosteroids, or anticoagulants with NSAIDs is one of the indicators applied to define the group at higher risk of NSAID-induced gastrointestinal toxicity regardless of doses. ${ }^{4}$ Therefore, patients with diabetes, who receive prophylactic aspirin, are inevitably at risk of both gastrointestinal bleeding (GIB) and CV events caused by NSAIDs.

However, beyond the risks from the unavoidable use of aspirin among patients with diabetes with high CV event risk, such patients are at further risk of negative $\mathrm{CV}$ outcomes from the use of NSAIDs other than aspirin. The safety profile of different NSAIDs among the group of those at high risk of CV events is not clear and difficult to 
study by clinical trial due to ethical issues. Furthermore, even though celecoxib was determined to be safe with regard to CV complications, unlike rofecoxib, ${ }^{5}$ the association between cyclooxygenase (COX) 2 selectivity and NSAID complications in real-world settings among those at high risk of $\mathrm{CV}$ events is an issue that requires further study. Moreover, NSAIDs known to have stronger COX-2 selectivity than celecoxib, such as meloxicam and nimesulide, as well as new COX-2 inhibitors such as etoricoxib, are currently on the market. There are earlier studies looking for an association of aspirin use with CV events among patients with and without diabetes, but none have evaluated the use of other NSAIDs. ${ }^{6} \quad 7$ Therefore, we conducted a cohort study on elderly patients with diabetes to assess GIB and CV outcomes of NSAID users compared to non-users, and to identify differences in the risk of each outcome associated with different kinds of NSAIDs.

\section{METHODS}

\section{Data source}

Ninety-seven percent of citizens of the Republic of Korea (hereafter, Korea) are covered by the national health insurance system and therefore have equal access to healthcare services. The Korean Health Insurance Review and Assessment Service (HIRA) database, which contains all the reimbursed claims of medication and medical procedures between January 2008 and December 2012 of the 50 million total residents of Korea, was used. This database contains demographics (age and sex), prescription data (brand and generic names of the drug; the amount, date, and duration of the prescription), and diagnosis information including death following the International Statistical Classification of Diseases and Related Health Problems, 10th Revision (ICD-10 codes). Since the present study used secondary healthcare data, it was exempted from review by the Institutional Review Board of Seoul National University Bundang Hospital (IRB No: X-1404/245-902) in April 2014.

\section{Study subjects and exposure assessments}

We identified new users and non-users of NSAIDs (Anatomical Therapeutic Chemical code M01A) among patients diagnosed with diabetes mellitus (ICD-10 code E10-14) in the claims database between 1 January 2008 and 31 December 2011. For NSAID users, the date of the first prescription for NSAIDs was considered the patient's index date, and those defined as NSAID non-users had to lack any NSAID prescription all throughout the study period of 2008-2012, and were assigned an index date corresponding to the same date of the matched NSAID user in order to minimize immortal time bias. Patients in both groups had to be at least 65 years old on the index date with no prescription of NSAIDs in the previous year to apply a new-user design in order to control for prevalent user bias such as underestimating early events and altering disease risk factors related to the study drugs. ${ }^{8}$ Therefore, NSAID users were selected from the year 2009 to 2011, and among these users, those who had received NSAID prescriptions in 2008 were excluded (figure 1). The risk of GIB among NSAID users is reported to decrease markedly 7 days after cessation, owing to the reversibility of the COX inhibition by NSAIDs. ${ }^{9}$ However, since elderly patients often receive NSAIDs occasionally, NSAID users were defined as patients with two or more prescriptions within 6 months from the index date with prescriptions within 30 days before the occurrence of an outcome, reflecting intermittent use with various gaps of treatment. The event rates during exposed and non-exposed periods in NSAID users and non-users were calculated applying exposed periods of users to the matched non-users. Multiple prescriptions of different NSAIDs were regarded as exposure to each different NSAID at that time when the risks of outcomes were compared among different kinds of NSAIDs. NSAID users and non-users were matched by using propensity scores applying predefined covariates that are related to both the exposure and outcome, since the model including only the surrogates for treatment has proved to imbalance propensity score matching. ${ }^{10}{ }^{11}$ Patients who had experienced GIB or CV events, cancer, trauma history, or coagulation disorders during 1 year prior to the index date were excluded to eliminate potential confounders to the association between NSAIDs and the outcomes. In Korea, one's diagnosis is cumulatively recorded in the claims database, making it difficult to determine whether or not the outcome is secondary. Therefore, patients who experienced either of the two outcomes of the study previously had to be excluded from the study subjects to detect the outcomes in the following years of follow-up without misclassification.

\section{Outcomes and covariates}

The two main outcomes of interest were GIB and CV events. GIB was defined as gastric, duodenal, peptic, or gastrojejunal ulcer with hemorrhage or perforation (ICD-10 code K250, K251, K252, K254, K255, K256, K260, K261, K262, K264, K265, K266, K270, K271, K272, K274, K275, K276, K280, K281, K282, K284, K285, K286), acute hemorrhagic gastritis (K290), hematemesis (K920), melena (K921), or unspecified GI hemorrhage (K922). CV events included acute myocardial infarction (I21) and ischemic stroke (I63). Outcomes were assessed from the index date until the last day of the study, assigning a range of 1-4 years of follow-up. Death was treated as a competing risk since it could happen before the outcome of interest in the elderly population. Only the first events were identified among multiple events, and when the two events occurred on the same day, they were both counted. Previous diagnosis of the following diseases was noted: hypertension, dyslipidemia, coronary artery disease, heart failure, atrial fibrillation/flutter, osteoarthritis, rheumatoid arthritis, liver disease, and renal failure. Recent use of the following medications was identified: antihypertensive 
Figure 1 Screening and enrollment of participants in the study (Gl, gastrointestinal; MI, myocardial infarction; NSAID, non-steroidal anti-inflammatory drug).

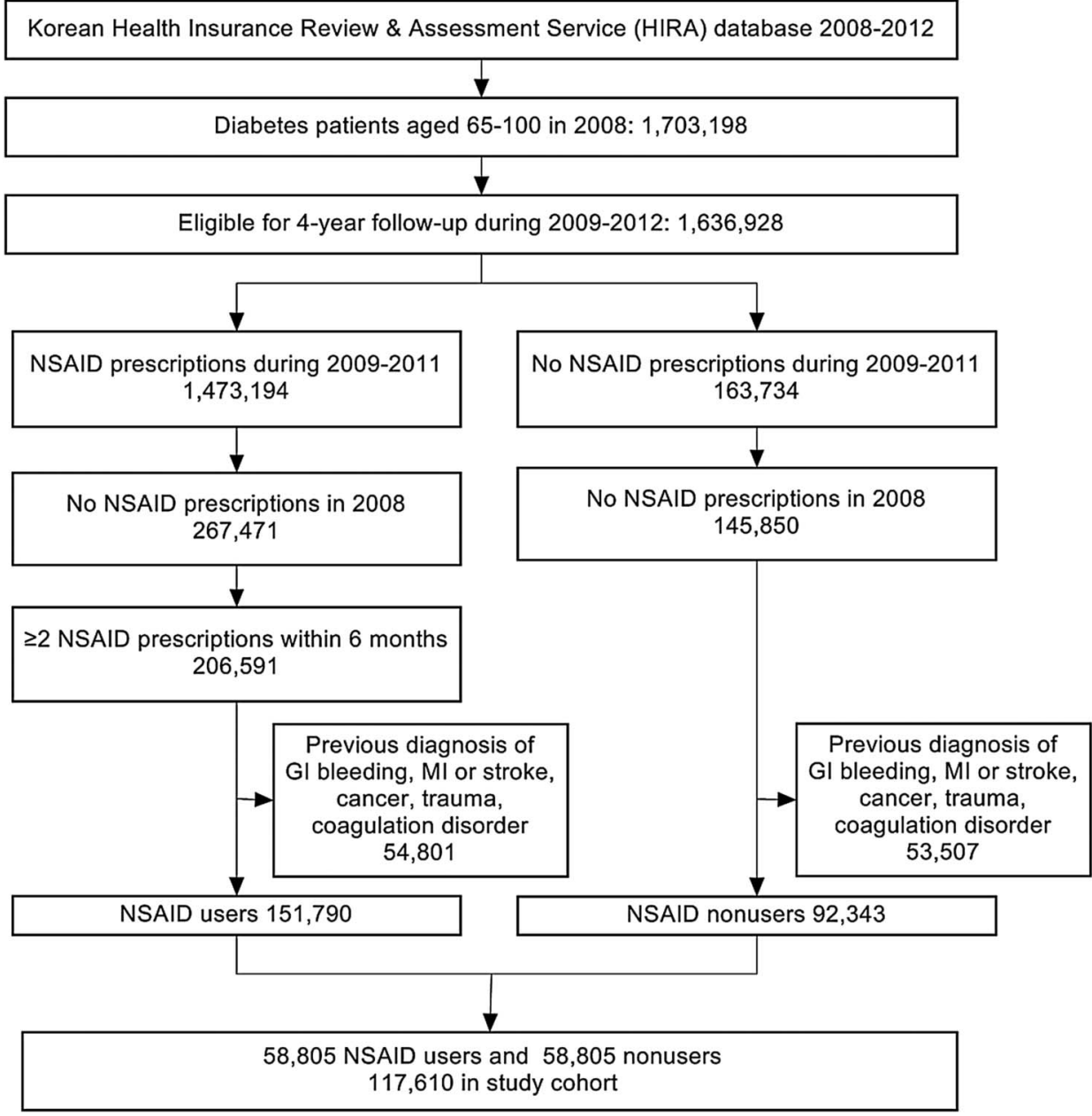

agents, anticoagulants, antiplatelet agents, aspirin, proton pump inhibitors (PPIs), gastroprotective agents (GPAs), corticosteroids, and selective serotonin reuptake inhibitors (SSRIs). In case of patients with diabetes who regularly visit clinics for their antidiabetics, NSAIDs including aspirin are generally obtained not over the counter but from their physicians due to their cheaper cost under national insurance coverage. Liver disease included alcoholic liver disease, toxic liver disease, hepatic failure, chronic hepatitis, and non-alcoholic fatty liver disease (K70, K71, K72, K73, K76), and renal failure included acute renal failure, chronic kidney disease, and unspecified kidney failure (N17, N18, N19). GPA included H2 receptor antagonists (H2RA), sucralfate, misoprostol, and rebamipide, the last of which is a cytoprotectant commonly used in Asian countries but not approved in the USA. Comorbidity was defined as having been diagnosed at least 1 year prior to the index date, and recent medication use was defined as having been prescribed no more than 1 year prior to the index date and during the follow-up period before the event occurred.

\section{Statistical analyses}

In order to select comparable participants from the NSAID user and non-user groups as well as from users of COX-2 selective inhibitors (coxibs) among NSAIDs, propensity score matching on a 1-to-1 basis was used. Covariates proven to be adequate for propensity score matching in the association between NSAIDs and upper gastrointestinal toxicity such as prior use of warfarin or oral steroids and a history of osteoarthritis, rheumatoid arthritis, hypertension, heart failure, and coronary artery disease were included on the basis of an earlier study, and other factors that are potential covariates in the relationship between NSAID use and CV events such as a history of dyslipidemia or atrial fibrillation, and previous use of aspirin, antiplatelets, and SSRIs were also included. ${ }^{11}$ The variables previously used to calculate the propensity score of selecting coxibs and NSAIDs were also considered. ${ }^{12}$ A greedy 5-to-1 digit matching algorithm without replacement was used to match NSAID users to non-users to reflect the situation in the real world. The balance after matching was evaluated by methods such as histogram plots, c-statistics, and the standardized differences between the two groups. Among these, standardized difference is known as the most effective way to measure balance considering differences $<10 \%$ to be well balanced. ${ }^{13}$ Also, the event rates during non-exposed periods in NSAID users and non-users using the exposed periods of the matched users were compared in order to clarify residual confounding after propensity score matching. We treated 
death as a competing risk to model the subdistribution hazard function. ${ }^{14}$ There were a total of 504 patients who died during the study period before the outcome occurred with an all-cause mortality of 4290 per million. The incidence rate (IR) with a 95\% CI per 1000 personyears and incidence rate ratio (IRR) of GIB and CV events were estimated. The crude and adjusted HRs (aHRs) and 95\% CIs of both outcomes were calculated for NSAID users versus non-users using univariate and multivariate Cox regression models. Time-dependent Cox regression was used regarding NSAID exposure as a time-varying covariate only in the case of CV events where the proportional hazards assumption was not met. The aHRs were adjusted for age, sex, comorbid conditions, and recent medications, all of which are listed in the tables. In the case of PPI and GPA users, early signs of GIB such as gastro-oesophageal reflux disease, peptic ulcer, gastritis, epigastric pain, or dyspepsia were additionally adjusted for to prevent protopathic bias. All statistical analyses were performed using SAS Enterprise Guide, V.6.1 (SAS Institute Inc, Cary, North Carolina, USA).

\section{RESULTS}

From the 1.7 million patients with diabetes aged over 65 years among the entire national population, 206591 patients were eligible to be in the NSAID user group and 145850 patients to be non-users. After following the exclusion criteria described previously, the two groups had 151790 and 92343 patients, respectively. By 1-to-1 propensity score matching, 58805 patients in each group were selected, comprising a total of 117610 study subjects (figure 1). Before propensity score matching, the NSAID users and non-users differed in age, comorbidities, and recent medications, while the propensity score-matched cohort resulted in a good balance in all characteristics according to histograms and standardized mean differences $<0.1$ with a c-statistic of 0.763 (table 1).

Within a total cohort of 117610 individuals, two outcomes, GIB and CV events such as myocardial infarction or stroke, were each assessed. The mean duration of follow-up time was 2.896 years (95\% CI 2.892 to 2.901) for GIB and 2.778 years (95\% CI 2.772 to 2.783) for CV events. During 4 years of follow-up, 2184 first episodes of GIB (1354 in NSAID users vs 830 in non-users) and 9333 episodes of myocardial infarction or stroke (5043 in NSAID users vs 4290 in non-users) were identified. In case of GIB, the event rate during exposed periods was 0.32 in NSAID users and 0.25 in non-users, while it was 0.68 and 0.75 , respectively, in users and non-users during non-exposed periods. In case of CV events, the event rates during exposure to NSAIDs in users and non-users were 0.27 and 0.23 , respectively, as against 0.73 and 0.77 in non-exposed periods. The IRR of GIB was 1.68 (95\% CI 1.54 to 1.84 ), and it was 1.21 (95\% CI 1.17 to 1.26 ) for $\mathrm{CV}$ events (tables 2 and 3). The number needed to harm was 111 for GIB and 77 for CV events.

The aHR of NSAID use was 1.68 (95\% CI 1.54 to 1.83 ) for GIB and 1.20 (95\% CI 1.15 to 1.25) for CV events. The risk of GIB was higher in patients with liver disease and renal failure and anticoagulant users (table 4). In the case of CV events, the risk was higher in patients with hypertension, coronary artery disease, and heart failure, as well as in anticoagulant users, antiplatelet agent users, aspirin users, and those treated with SSRIs. Among study subjects, the most commonly prescribed NSAIDs were loxoprofen sodium, aceclofenac, talniflumate, and dexibuprofen consisting of $69.1 \%$ of the total NSAIDs (see online supplementary table S1). However, compared to celecoxib users, nimesulide increased the risk of GIB and ketorolac increased the risk of CV events (aHR 2.60 and 3.13, respectively) (see online supplementary table S2).

\section{DISCUSSION}

Even though coxibs are known to have a low risk of GIB among NSAIDs, their CV risk must also be taken into account. However, diabetes is a well-known risk factor of CV diseases, and thus patients with diabetes often receive prophylactic aspirin, which increases the risk of GIB. The purpose of this study was to evaluate the risk of both GIB and CV outcomes among patients with diabetes, comparing NSAID users to non-users, and also to discover different risks among different NSAIDs. All of the IRRs of GIB and CV events exceeded 1 and were at least marginally significant among aged patients with diabetes with various comorbidities and recent medications, indicating a higher risk of both outcomes within patients with diabetes, except for CV events in patients with rheumatoid arthritis where the IRR was not significant due to the small number of patients in this group. This implies the need to assess the additional risk of both CV events and GIB in patients with diabetes who were being prescribed NSAIDs.

The risk of GIB was higher among patients with liver disease and renal failure. Patients with cirrhosis may develop GIB resulting from esophageal or gastric varices. However, patients with non-cirrhosis liver disease as defined in this study, who probably would have no varices, were also vulnerable to GIB, which indicates that patients with liver disease have a higher risk of nonvariceal GIB when treating NSAIDs. Also, in another study, patients with hepatic disease and renal disease had a higher risk of upper gastrointestinal complications from peptic ulcer disease caused by NSAIDs. ${ }^{15}$ However, patients with end-stage renal disease are documented to have a higher risk of peptic ulcer bleeding owing to mechanisms such as platelet dysfunction and blood coagulation abnormalities, ${ }^{16}$ and acute renal failure is also proposed to be associated with upper GIB. ${ }^{17}$ According to the ACG Guideline, users from the elderly population of any one of anticoagulants, aspirin, and 
Table 1 Study subjects before and after propensity score matching

\begin{tabular}{|c|c|c|c|c|c|c|c|c|c|c|}
\hline \multirow[b]{3}{*}{ Characteristics } & \multicolumn{5}{|c|}{ Initial cohort } & \multicolumn{5}{|c|}{ 1:1 Propensity score-matched cohort } \\
\hline & \multicolumn{2}{|c|}{ NSAID users } & \multicolumn{2}{|c|}{$\begin{array}{l}\text { NSAID } \\
\text { non-users }\end{array}$} & \multirow[b]{2}{*}{$\mathbf{d}^{*}$} & \multicolumn{2}{|c|}{ NSAID users } & \multicolumn{2}{|c|}{$\begin{array}{l}\text { NSAID } \\
\text { non-users }\end{array}$} & \multirow[b]{2}{*}{$\mathbf{d}^{*}$} \\
\hline & $\mathbf{N}$ & (\%) & $\mathbf{N}$ & $(\%)$ & & $\overline{\mathbf{N}}$ & $(\%)$ & $\mathbf{N}$ & $(\%)$ & \\
\hline Total & 151790 & & 92343 & & & 58805 & & 58805 & & \\
\hline Male sex & 67390 & 44.4 & 43668 & 47.3 & 0.058 & 28557 & 48.6 & 28819 & 49.0 & 0.008 \\
\hline Age, mean (SD), years & \multicolumn{2}{|c|}{$73.06(5.90)$} & \multicolumn{2}{|c|}{$74.47(7.19)$} & 0.214 & \multicolumn{2}{|c|}{$74.38(6.64)$} & \multicolumn{2}{|c|}{$74.27(6.70)$} & \\
\hline \multicolumn{11}{|l|}{ Age group, years } \\
\hline $65-69$ & 52886 & 34.8 & 29197 & 31.6 & 0.068 & 17458 & 29.7 & 18919 & 32.2 & 0.054 \\
\hline $70-74$ & 47073 & 31.0 & 22664 & 24.5 & 0.146 & 16079 & 27.3 & 14848 & 25.3 & 0.045 \\
\hline $75-79$ & 28793 & 19.0 & 17428 & 18.9 & 0.003 & 11739 & 20.0 & 11214 & 19.1 & 0.023 \\
\hline $80+$ & 23038 & 15.2 & 23054 & 25.0 & 0.246 & 13529 & 23.0 & 13824 & 23.5 & 0.012 \\
\hline \multicolumn{11}{|l|}{ Previous diagnosis } \\
\hline Hypertension & 98023 & 64.6 & 61535 & 66.6 & 0.042 & 38478 & 65.4 & 38574 & 65.6 & 0.004 \\
\hline Dyslipidemia & 66642 & 43.9 & 34289 & 37.1 & 0.139 & 22565 & 38.4 & 22434 & 38.2 & 0.006 \\
\hline Coronary artery disease & 11058 & 7.3 & 7270 & 7.9 & 0.023 & 4788 & 8.1 & 4532 & 7.7 & 0.015 \\
\hline Heart failure & 6491 & 4.3 & 5904 & 6.4 & 0.093 & 3690 & 6.3 & 3609 & 6.1 & 0.008 \\
\hline Atrial fibrillation/flutter & 3572 & 2.4 & 1987 & 2.2 & 0.013 & 1433 & 2.4 & 1295 & 2.2 & 0.013 \\
\hline Osteoarthritis & 43011 & 28.3 & 10964 & 11.9 & 0.418 & 8027 & 13.7 & 7681 & 13.1 & 0.018 \\
\hline Rheumatoid arthritis & 3829 & 2.5 & 945 & 1.0 & 0.115 & 817 & 1.4 & 690 & 1.2 & 0.057 \\
\hline Liver disease & 31483 & 20.7 & 14601 & 15.8 & 0.127 & 10574 & 18.0 & 10149 & 17.3 & 0.018 \\
\hline Renal failure & 4770 & 3.1 & 5444 & 5.9 & 0.135 & 3269 & 5.6 & 3146 & 5.4 & 0.013 \\
\hline \multicolumn{11}{|l|}{ Medication use } \\
\hline Antihypertensive agent & 105773 & 69.7 & 63181 & 68.4 & 0.028 & 41440 & 70.5 & 41759 & 71.0 & 0.011 \\
\hline Anticoagulant & 12553 & 8.3 & 7893 & 8.6 & 0.007 & 5531 & 9.4 & 5305 & 9.0 & 0.014 \\
\hline Antiplatelet agent & 24665 & 16.3 & 13304 & 14.4 & 0.050 & 9157 & 15.6 & 8988 & 15.3 & 0.008 \\
\hline Aspirin & 57896 & 38.1 & 33390 & 36.2 & 0.039 & 21988 & 37.4 & 21770 & 37.0 & 0.008 \\
\hline Proton pump inhibitor & 14768 & 9.7 & 5543 & 6.0 & 0.138 & 4132 & 7.0 & 3845 & 6.6 & 0.020 \\
\hline Gastroprotective agent & 112526 & 74.1 & 39944 & 43.3 & 0.659 & 29521 & 50.0 & 29311 & 50.0 & 0.004 \\
\hline Corticosteroid & 45406 & 30.0 & 14468 & 15.7 & 0.343 & 10826 & 18.4 & 10549 & 18.0 & 0.010 \\
\hline SSRI & 10647 & 7.0 & 4787 & 5.2 & 0.075 & 3667 & 6.2 & 3396 & 5.8 & 0.017 \\
\hline
\end{tabular}

*Standardized differences.

NSAID, non-steroidal anti-inflammatory drug; SSRI, selective serotonin reuptake inhibitor.

corticosteroids are classified as a group at high risk of NSAID toxicity, while the aHR of GIB was higher only in anticoagulant users. Antiplatelet agents can give rise to GIB by producing ulcers and erosions, while anticoagulants might precipitate bleeding from pre-existing lesions. Even though the preventive effect of PPI or GPA in GIB is well known among NSAID and aspirin users from a variety of studies, it did not show statistical significance. ${ }^{18}$ In fact, among 7977 patients who received PPI, a total of $67 \%$ were prescribed with at least one of the medications among anticoagulants, aspirin, and corticosteroids. Thus, PPI users eventually became more vulnerable to NSAID toxicity and showed a non-significance in GIB despite the prevention of protopathic bias by adjustment of early symptoms of GIB such as gastritis and epigastric pain.

When there are high risks of $\mathrm{CV}$ diseases or GIB, the ACG Guideline recommends prescribing PPI with NSAIDs or choosing COX-2 inhibitors. Since the study population is elderly patients with diabetes who are certainly at high risk of both events, the protective effects of the two treatments were compared. Among NSAID users, the aHR of celecoxib use was 1.09 (95\% CI 0.79 to 1.50$)$ while that of PPI + NSAID use was $1.10(95 \%$ CI 0.93 to 1.31 ) (data not shown). In other words, the risks of GIB in celecoxib users and PPI + NSAID users were similar, with overlapping CIs without substantial protective effect. While the CONDOR (celecoxib vs omeprazole and diclofenac in patients with osteoarthritis and rheumatoid arthritis) study found results in celecoxib's favor, the main cause behind this result was anemia, whereas the rates of actual upper GIB did not differ between the two groups of celecoxib and PPI + NSAID. ${ }^{19}$ This could explain the similar risk between the two groups in this study since each event was counted from the diagnosis of individuals.

Even though aspirin is known to increase GIB risk by direct injury to the gastrointestinal mucosa, this group did not show a significant GIB risk after adjustment. Furthermore, in a former study, while the baseline risk of GIB in the absence of aspirin was higher among patients with diabetes, the use of aspirin was associated with a greater GIB risk only among individuals without diabetes, ${ }^{6}$ and the benefit of aspirin was reported to be similar in patients with and without diabetes in a meta-analysis of primary prevention of $\mathrm{CV}$ events. ${ }^{20}$ The 
Table 2 IRs of upper gastrointestinal bleeding by sample characteristics

\begin{tabular}{|c|c|c|c|c|c|c|}
\hline \multirow[b]{4}{*}{ Subgroups } & \multicolumn{6}{|c|}{ Upper gastrointestinal bleeding } \\
\hline & \multicolumn{4}{|c|}{ Incidence rate per 1000 person-years } & \multirow[b]{3}{*}{ IRR } & \multirow[b]{3}{*}{$(95 \% \mathrm{Cl})$} \\
\hline & \multicolumn{2}{|c|}{ NSAID users } & \multicolumn{2}{|c|}{ NSAIDs non-users } & & \\
\hline & $\mathbf{I R}$ & $(95 \% \mathrm{Cl})$ & $\mathbf{I R}$ & $(95 \% \mathrm{Cl})$ & & \\
\hline Entire sample & 8.11 & (8.10 to 8.13$)$ & 4.82 & (4.81 to 4.83 ) & 1.68 & (1.54 to 1.84$)$ \\
\hline \multicolumn{7}{|l|}{ Gender } \\
\hline Male & 9.18 & (9.16 to 9.20$)$ & 5.25 & (5.24 to 5.27$)$ & 1.75 & (1.55 to 1.97$)$ \\
\hline Female & 7.12 & (7.10 to 7.14$)$ & 4.41 & (4.40 to 4.42$)$ & 1.62 & (1.42 to 1.83$)$ \\
\hline \multicolumn{7}{|l|}{ Age group, years } \\
\hline $65-69$ & 7.51 & (7.49 to 7.54$)$ & 4.55 & (4.54 to 4.57$)$ & 1.65 & (1.41 to 1.93$)$ \\
\hline $70-74$ & 8.04 & (8.01 to 8.06$)$ & 4.82 & (4.81 to 4.84$)$ & 1.67 & (1.41 to 1.98$)$ \\
\hline $75-79$ & 8.99 & (8.95 to 9.02$)$ & 5.45 & (5.43 to 5.47$)$ & 1.65 & (1.37 to 1.99$)$ \\
\hline $80-$ & 8.25 & (8.22 to 8.28$)$ & 4.68 & (4.66 to 4.70$)$ & 1.76 & (1.47 to 2.11$)$ \\
\hline \multicolumn{7}{|l|}{ Previous diagnosis } \\
\hline Hypertension & 8.36 & (8.34 to 8.38$)$ & 4.75 & (4.74 to 4.76$)$ & 1.76 & (1.58 to 1.96$)$ \\
\hline Dyslipidemia & 8.56 & (8.54 to 8.59 ) & 4.92 & (4.91 to 4.94$)$ & 1.74 & (1.52 to 2.00$)$ \\
\hline Coronary artery disease & 8.72 & (8.67 to 8.77 ) & 5.88 & (5.85 to 5.92$)$ & 1.48 & (1.12 to 1.97$)$ \\
\hline Heart failure & 11.27 & (11.21 to 11.34$)$ & 5.13 & (5.10 to 5.18$)$ & 2.20 & (1.60 to 3.02 ) \\
\hline Atrial fibrillation/flutter & 10.32 & (10.22 to 10.42$)$ & 6.40 & (6.34 to 6.48$)$ & 1.61 & (0.98 to 2.65$)$ \\
\hline Osteoarthritis & 8.65 & (8.61 to 8.69 ) & 4.80 & (4.78 to 4.83 ) & 1.80 & (1.43 to 2.27$)$ \\
\hline Rheumatoid arthritis & 12.94 & (12.80 to 13.09 ) & 3.62 & (3.57 to 3.70 ) & 3.57 & (1.64 to 7.79 ) \\
\hline Chronic liver disease & 9.52 & (9.49 to 9.56$)$ & 5.88 & (5.86 to 5.90$)$ & 1.62 & (1.35 to 1.95$)$ \\
\hline Chronic renal disease & 15.39 & (15.31 to 15.47 ) & 5.89 & (5.86 to 5.94$)$ & 2.61 & (1.91 to 3.57 ) \\
\hline \multicolumn{7}{|l|}{ Medication use } \\
\hline Antihypertensive agent & 8.43 & (8.41 to 8.44$)$ & 4.87 & (4.86 to 4.89$)$ & 1.73 & (1.56 to 1.91$)$ \\
\hline Anticoagulant & 12.15 & (12.10 to 12.21$)$ & 5.55 & (5.52 to 5.58$)$ & 2.19 & (1.70 to 2.82$)$ \\
\hline Antiplatelet agent & 9.32 & (9.28 to 9.35$)$ & 5.42 & (5.40 to 5.45$)$ & 1.72 & (1.40 to 2.11$)$ \\
\hline Aspirin & 8.89 & (8.87 to 8.91$)$ & 4.97 & (4.96 to 4.99$)$ & 1.79 & (1.56 to 2.05 ) \\
\hline Proton pump inhibitor & 14.23 & (14.16 to 14.30$)$ & 6.82 & (6.78 to 6.86$)$ & 2.09 & (1.60 to 2.73 ) \\
\hline Gastroprotective agent & 9.26 & (9.24 to 9.28$)$ & 5.78 & (5.77 to 5.80$)$ & 1.60 & (1.43 to 1.79$)$ \\
\hline Corticosteroid & 9.68 & (9.64 to 9.71$)$ & 4.87 & (4.85 to 4.89$)$ & 1.99 & (1.64 to 2.41$)$ \\
\hline SSRI & 9.57 & (9.51 to 9.63 ) & 4.36 & (4.33 to 4.40$)$ & 2.20 & (1.55 to 3.12$)$ \\
\hline
\end{tabular}

IR, incidence rate; IRR, incidence rate ratio; NSAID, non-steroidal anti-inflammatory drug; SSRI, selective serotonin reuptake inhibitor.

inter-individual variability in drug response or inadequate thromboxane inhibition by aspirin, known as "resistance," due to the variable turnover of the thromboxane in platelets could describe this, which may be related to glycemic control in patients with diabetes. ${ }^{21} 22$ However, the inhibitory effect of aspirin by other NSAIDs is not likely in this study since recent medication use was detected during the follow-up period before the event occurred and also before the last prescription of NSAIDs. In another study, the relative risk of upper GIB in patients taking aspirin was higher when used for primary prevention than for secondary prevention, but this difference was more than compensated for by the lower baseline risk in the primary prevention population. Therefore, since aspirin was used for primary prevention of $\mathrm{CV}$ events in this study, the protective effect from GIB of aspirin could have been concealed.

The higher risk of CV events among those with coronary artery disease or heart failure and users of anticoagulants, antiplatelet agents, or even aspirin use can be explained by the higher probability of developing myocardial infarction or stroke among these groups. However, even though aspirin is a well-known primary prevention strategy in those with diabetes at increased CV risk, it resulted in a higher risk of CV events. Among 43758 aspirin users, $83.4 \%$ were diagnosed with hypertension, $91 \%$ were being prescribed antihypertensive medication, and $53.5 \%$ were patients with dyslipidemia, who are inevitably considered to be a high-risk group for CV events, especially when combined with diabetes. Thus, confounding by indication is another explanation. However, SSRI users had an unexpectedly high risk of a CV event, while the well-known GIB risk was not shown. An earlier study reported the beneficial evidence of SSRIs in CV events, ${ }^{23}$ whereas another study did so only with long-term use. ${ }^{24}$ However, depression itself is regarded as a cause of coronary heart disease by two mechanisms: behavioral factors (physical activity, smoking, and non-compliance) and biological factors (CV autonomic dysregulation and inflammation). ${ }^{25}$ In another study, hypoglycemia was associated with depressive symptoms. ${ }^{26}$ Furthermore, a recent meta-analysis finding reported an increased risk of ischemic or hemorrhagic stroke by SSRIs independent of depression, and suggested vasoconstriction in cerebral arteries caused by SSRIs as a possible reason. ${ }^{27}$ Likewise, SSRIs were found 
Table 3 Incidence rates of myocardial infarction or ischemic stroke by sample characteristics

\begin{tabular}{|c|c|c|c|c|c|c|}
\hline \multirow[b]{4}{*}{ Subgroups } & \multicolumn{6}{|c|}{ Myocardial infarction or ischemic stroke } \\
\hline & \multicolumn{4}{|c|}{ Incidence rate per 1000 person-years } & \multirow[b]{3}{*}{ IRR } & \multirow[b]{3}{*}{$(95 \% \mathrm{Cl})$} \\
\hline & \multicolumn{2}{|c|}{ NSAID users } & \multicolumn{2}{|c|}{ NSAIDs non-users } & & \\
\hline & $\overline{I R}$ & $(95 \% \mathrm{Cl})$ & $\mathbf{I R}$ & $(95 \% \mathrm{Cl})$ & & \\
\hline Entire sample & 31.54 & (31.52 to 31.57$)$ & 25.98 & (25.96 to 26.01$)$ & 1.21 & (1.17 to 1.26$)$ \\
\hline \multicolumn{7}{|l|}{ Gender } \\
\hline Male & 33.84 & (33.80 to 33.88$)$ & 27.14 & (27.11 to 27.17$)$ & 1.25 & (1.18 to 1.32$)$ \\
\hline Female & 29.42 & (29.38 to 29.46$)$ & 24.88 & (24.86 to 24.92$)$ & 1.18 & (1.12 to 1.25$)$ \\
\hline \multicolumn{7}{|l|}{ Age group, years } \\
\hline $65-69$ & 22.46 & (22.42 to 22.50$)$ & 20.06 & (20.03 to 20.09) & 1.12 & (1.03 to 1.22$)$ \\
\hline $70-74$ & 30.41 & (30.36 to 30.46$)$ & 28.70 & (28.66 to 28.75 ) & 1.06 & (0.98 to 1.15$)$ \\
\hline $75-79$ & 38.67 & (38.60 to 38.73 ) & 30.89 & (30.85 to 30.96$)$ & 1.25 & (1.15 to 1.36$)$ \\
\hline $80-$ & 39.24 & (39.18 to 39.31 ) & 27.43 & (27.40 to 27.48 ) & 1.43 & (1.32 to 1.55$)$ \\
\hline \multicolumn{7}{|l|}{ Previous diagnosis } \\
\hline Hypertension & 33.65 & (33.61 to 33.68 ) & 27.36 & (27.34 to 27.39 ) & 1.23 & (1.17 to 1.29$)$ \\
\hline Dyslipidemia & 30.67 & (30.63 to 30.72 ) & 25.78 & (25.75 to 25.82$)$ & 1.19 & (1.11 to 1.27$)$ \\
\hline Coronary artery disease & 42.63 & (42.51 to 42.74 ) & 34.31 & (34.23 to 34.41$)$ & 1.24 & (1.10 to 1.41$)$ \\
\hline Heart failure & 41.95 & (41.82 to 42.08 ) & 33.00 & (32.92 to 33.11 ) & 1.27 & (1.10 to 1.47 ) \\
\hline Atrial fibrillation/flutter & 43.34 & (43.13 to 43.55$)$ & 26.01 & (25.89 to 26.17 ) & 1.67 & (1.30 to 2.14$)$ \\
\hline Osteoarthritis & 34.15 & (34.07 to 34.23 ) & 28.85 & (28.80 to 28.92$)$ & 1.18 & (1.07 to 1.31 ) \\
\hline Rheumatoid arthritis & 28.37 & (28.15 to 28.59 ) & 23.14 & (22.99 to 23.34 ) & 1.23 & (0.85 to 1.78$)$ \\
\hline Chronic liver disease & 29.07 & (29.01 to 29.13 ) & 23.06 & (23.02 to 23.11 ) & 1.26 & (1.14 to 1.39$)$ \\
\hline Chronic renal disease & 40.74 & (40.60 to 40.87 ) & 22.85 & (22.78 to 22.95 ) & 1.78 & (1.50 to 2.12$)$ \\
\hline \multicolumn{7}{|l|}{ Medication use } \\
\hline Antihypertensive agent & 33.19 & (33.16 to 33.22 ) & 27.15 & (27.12 to 27.17 ) & 1.22 & (1.17 to 1.28$)$ \\
\hline Anticoagulant & 42.20 & (42.10 to 42.31 ) & 30.31 & (30.25 to 30.40 ) & 1.39 & (1.23 to 1.57$)$ \\
\hline Antiplatelet agent & 45.64 & (45.56 to 45.73 ) & 39.16 & (39.11 to 39.24$)$ & 1.17 & (1.07 to 1.27 ) \\
\hline Aspirin & 34.49 & (34.45 to 34.54$)$ & 29.77 & (29.74 to 29.81 ) & 1.16 & (1.09 to 1.23 ) \\
\hline Proton pump inhibitor & 34.36 & (34.25 to 34.47 ) & 26.32 & (26.25 to 26.42$)$ & 1.31 & (1.12 to 1.52$)$ \\
\hline Gastroprotective agent & 33.44 & (33.40 to 33.48 ) & 28.27 & (28.25 to 28.31 ) & 1.18 & (1.12 to 1.25$)$ \\
\hline Corticosteroid & 33.42 & (33.35 to 33.49 ) & 26.33 & (26.29 to 26.39 ) & 1.27 & (1.16 to 1.39$)$ \\
\hline SSRI & 38.77 & (38.65 to 38.89 ) & 31.60 & (31.52 to 31.71 ) & 1.23 & (1.06 to 1.42$)$ \\
\hline
\end{tabular}

IR, incidence rate; IRR, incidence rate ratio; NSAID, non-steroidal anti-inflammatory drug; SSRI, selective serotonin reuptake inhibitor.

to increase arterial stiffness in the elderly, ${ }^{28}$ and use of an SSRI was associated with increases in the heart rate and decreases in its variability. ${ }^{29}$

When each was compared to celecoxib, nimesulide increased the risk of GIB and ketorolac increased the risk of $\mathrm{CV}$ events. Nimesulide is a COX-2 selective NSAID and manifested a coxib-like benefit in another study, ${ }^{30}$ but this has not been consistent, showing a higher risk of GIB than other non-selective NSAIDs in another study. ${ }^{31}$ It is not approved in the USA and was withdrawn in many countries due to its hepatotoxicity. In 2011, the European Medicines Agency made a recommendation that nimesulide be prescribed in acute pain or dysmenorrhea but not in osteoarthritis. However, it is still on the market in Korea as in a few other countries, and $1.6 \%$ of the GIB cohort and $2.2 \%$ of the CV cohort received it, which corresponds well with $2 \%$ use among orthopedic patients in Korea in another study. ${ }^{32}$ Even though it could have been prescribed to patients with a higher risk of GIB, thus resulting in a higher risk in this study, lack of evidence on safety so far and the higher risk of GIB found in this study triggers an alert against the use of nimesulide. The CV risk of ketorolac, a non- selective NSAID, was marginally higher than that of other NSAIDs in this study, which is similar to the findings of other studies both in cases of myocardial infarction $^{33}$ and ischemic stroke, ${ }^{34}$ with ORs of 2.02 and 1.90 , respectively. Naproxen may be the agent of choice among patients with high CV risk, but only $1.8 \%$ among the cohort was found to have received it. Specifically, prescribing NSAIDs only by the COX-2 selectivity determined in vitro by the IC50 ratios of COX-1 and COX-2 should be avoided since COX-2 selectivity is not directly correlated with risky outcomes in real life. ${ }^{35}$ This could be explained by the kinetic diversity in the COX inhibition mechanism, with which IC50 values are not related, and the COX-independent physiochemical properties of NSAIDs. ${ }^{36}$ This also means that the current hypothesis of unbalance in thromboxane A2 (TXA2) and prostacyclin (PGI2) as the mechanism of $\mathrm{CV}$ risk of coxibs might not be a possible explanation for elderly patients with diabetes. ${ }^{37}$

This study has some limitations. First, the claims database does not include diagnostic laboratory findings, a family history, or lifestyle factors such as smoking or obesity. Therefore, the reason for higher risk of CV 
Table 4 HRs of upper gastrointestinal bleeding and cardiovascular events

\begin{tabular}{|c|c|c|c|c|c|c|c|c|}
\hline & \multicolumn{4}{|c|}{ Gastrointestinal bleeding } & \multicolumn{4}{|c|}{ Cardiovascular events } \\
\hline & cHR & (95\% Cl) & aHR & $(95 \% \mathrm{Cl})$ & cHR & $(95 \% \mathrm{Cl})$ & aHR & $(95 \% \mathrm{Cl})$ \\
\hline Entire sample & 1.68 & (1.54 to 1.83$)$ & 1.68 & (1.54 to 1.83$)$ & 1.21 & (1.16 to 1.26$)$ & 1.20 & (1.15 to 1.25$)$ \\
\hline \multicolumn{9}{|l|}{ Gender } \\
\hline Male & 1.25 & (1.15 to 1.36$)$ & 1.28 & (1.17 to 1.40$)$ & 1.12 & (1.07 to 1.16$)$ & 1.20 & (1.15 to 1.26$)$ \\
\hline Female & 1.00 & & 1.00 & & 1.00 & & 1.00 & \\
\hline \multicolumn{9}{|l|}{ Age group, years } \\
\hline $65-69$ & 1.00 & & 1.00 & & 1.00 & & 1.00 & \\
\hline $70-74$ & 1.08 & (0.96 to 1.20$)$ & 1.04 & (0.93 to 1.16$)$ & 1.38 & (1.30 to 1.46$)$ & 1.35 & (1.27 to 1.43$)$ \\
\hline $75-79$ & 1.20 & (1.07 to 1.35$)$ & 1.18 & (1.05 to 1.33$)$ & 1.62 & (1.52 to 1.72$)$ & 1.58 & (1.49 to 1.68$)$ \\
\hline $80-$ & 1.06 & (0.94 to 1.19$)$ & 1.07 & (0.95 to 1.21$)$ & 1.53 & (1.45 to 1.62$)$ & 1.52 & (1.43 to 1.62$)$ \\
\hline \multicolumn{9}{|l|}{ Previous diagnosis } \\
\hline Hypertension & 1.03 & (0.95 to 1.13$)$ & 0.96 & (0.85 to 1.08$)$ & 1.19 & to 1.24$)$ & 1.11 & $(1.05$ \\
\hline Dyslipidemia & 1.07 & (0.98 to 1.17$)$ & 0.99 & (0.90 to 1.09$)$ & 0.97 & (0.93 to 1.01$)$ & 0.91 & (0.87 to 0.95$)$ \\
\hline Coronary artery disease & 1.15 & (0.99 to 1.33 ) & 0.97 & (0.83 to 1.14$)$ & 1.38 & (1.30 to 1.48$)$ & 1.13 & (1.05 to 1.22$)$ \\
\hline Heart failure & 1.28 & (1.10 to 1.50$)$ & 1.10 & (0.93 to 1.29$)$ & 1.33 & (1.23 to 1.43$)$ & 1.11 & (1.03 to 1.21$)$ \\
\hline Atrial fibrillation/flutter & 1.32 & (1.04 to 1.69$)$ & 1.12 & (0.88 to 1.43$)$ & 1.23 & (1.08 to 1.39$)$ & 1.10 & (0.97 to 1.24$)$ \\
\hline Osteoarthritis & 1.06 & (0.94 to 1.19$)$ & 1.02 & (0.90 to 1.16$)$ & 1.12 & (1.06 to 1.19$)$ & 1.05 & (0.99 to 1.12$)$ \\
\hline Rheumatoid arthritis & 1.32 & (0.96 to 1.82$)$ & 1.21 & (0.87 to 1.67$)$ & 0.91 & (0.76 to 1.10$)$ & 0.86 & (0.72 to 1.04$)$ \\
\hline Liver disease & 1.25 & (1.13 to 1.39$)$ & 1.16 & (1.04 to 1.29$)$ & 0.89 & (0.85 to 0.95$)$ & 0.90 & (0.85 to 0.95$)$ \\
\hline Renal failure & 1.67 & (1.44 to 1.94$)$ & 1.39 & (1.19 to 1.62$)$ & 1.09 & (1.01 to 1.19$)$ & 0.92 & (0.84 to 1.00$)$ \\
\hline \multicolumn{9}{|l|}{ Medication use } \\
\hline Antihypertensive agent & 1.10 & 0 1.21) & 1.02 & & 18 & & 0.96 & $.03)$ \\
\hline Anticoagulant & 1.42 & (1.25 to 1.61$)$ & 1.20 & (1.05 to 1.38$)$ & 1.29 & (1.21 to 1.37 ) & 1.11 & (1.04 to 1.19$)$ \\
\hline Antiplatelet agent & 1.17 & (1.05 to 1.30$)$ & 1.02 & (0.91 to 1.15$)$ & 1.61 & (1.53 to 1.69$)$ & 1.49 & (1.41 to 1.57$)$ \\
\hline Aspirin & 1.12 & (1.02 to 1.22$)$ & 1.07 & (0.97 to 1.17$)$ & 1.20 & (1.15 to 1.25$)$ & 1.11 & (1.06 to 1.16$)$ \\
\hline Proton pump inhibitor & 1.72 & (1.50 to 1.96$)$ & 0.96 & (0.86 to 1.08$)$ & 1.07 & (0.99 to 1.16$)$ & 0.91 & (0.85 to 0.97$)$ \\
\hline Gastroprotective agent & 1.40 & (1.29 to 1.52$)$ & 0.98 & (0.89 to 1.07$)$ & 1.17 & (1.12 to 1.22$)$ & 0.89 & (0.86 to 0.93 ) \\
\hline Corticosteroid & 1.16 & (1.04 to 1.28$)$ & 1.01 & (0.91 to 1.06$)$ & 1.05 & (1.00 to 1.11 ) & 0.96 & (0.91 to 1.02 ) \\
\hline SSRI & 1.10 & (0.93 to 1.30$)$ & 0.98 & (0.83 to 1.17$)$ & 1.25 & (1.16 to 1.36 ) & 1.16 & (1.07 to 1.25$)$ \\
\hline
\end{tabular}

aHR, adjusted HR; cHR, crude HR; IR, incidence rate; IRR, incidence rate ratio; NSAID, non-steroidal anti-inflammatory drug; SSRI, selective serotonin reuptake inhibitor.

events in aspirin users cannot be interpreted as the risk of the medication itself, but as an indicator of a high-risk group since these prescribers must have a clinical background for prescription and the drugs were being prescribed for reduction of potential CV risk, which was not possible to detect in the claims database. Nevertheless, one study showed that a failure to adjust a few potential confounders did not cause notable bias in an example of coxibs and myocardial infarction. ${ }^{38}$ Moreover, it was reported in another study that non-gastrointestinal comorbidity is an independent risk factor for GIB and contributes even more than aspirin or NSAIDs. ${ }^{39}$ Thus, we used various relevant comorbidities in generating propensity scores for matching NSAID users to nonusers and adjusting the Cox regression models. Second, we identified outcomes and comorbidities by ICD-10 codes. However, in order to increase the validity, the codes were used throughout four digits in detecting GIB events, and outcomes such as myocardial infarction or ischemic stroke are regarded as difficult to misdiagnose. Third, NSAIDs including aspirin are frequently obtained over the counter. However, due to the national health insurance system in Korea, patients receive most of their medication including aspirin or NSAIDs through prescriptions from clinicians since that is cheaper than purchasing over-the-counter drugs making it possible to detect the use of aspirin or NSAIDs in the claims data. Therefore, misclassification is unlikely to be significant.

\section{CONCLUSIONS}

In conclusion, elderly patients with diabetes using NSAIDs were at a higher risk of both GIB and CV events compared to non-users, and these risks differed among different NSAIDs in a way that did not correspond directly to COX-2 selectivity.

Acknowledgements The authors thank Jocelyn Graf from Proficia for English proofreading.

Contributors JK designed the study, managed the data, and drafted the manuscript. JL designed the study and contributed to the statistical analysis and discussion. CMS contributed to the study design and discussion. DHL and B-JP contributed to the design of the study and reviewed the manuscript. $\mathrm{DHL}$ and B-JP are the guarantors of this work and take identical responsibility for the integrity of the data and the accuracy of the data analysis.

Funding The data for analysis were provided by the Health Insurance Review \& Assessment Service, Ministry of Health and Welfare and the study was supported by grant no Z-2014-22 from the Seoul National University Bundang Hospital Research fund. 
Competing interests None declared.

Ethics approval Seoul National University Bundang Hospital.

Provenance and peer review Not commissioned; internally peer reviewed.

Data sharing statement No additional data are available.

Open Access This is an Open Access article distributed in accordance with the Creative Commons Attribution Non Commercial (CC BY-NC 4.0) license, which permits others to distribute, remix, adapt, build upon this work noncommercially, and license their derivative works on different terms, provided the original work is properly cited and the use is non-commercial. See: http:// creativecommons.org/licenses/by-nc/4.0/

\section{REFERENCES}

1. Bhala N, Emberson J, Merhi A, et al, Coxib traditional Nsaid Trialists' Collaboration. Vascular and upper gastrointestinal effects of non-steroidal anti-inflammatory drugs: meta-analyses of individual participant data from randomised trials. Lancet 2013;382:769-79.

2. Weil J, Langman MJ, Wainwright $P$, et al. Peptic ulcer bleeding: accessory risk factors and interactions with non-steroidal anti-inflammatory drugs. Gut 2000;46:27-31.

3. American Diabetes A. Standards of medical care in diabetes-2014. Diabetes Care 2014;37(Suppl 1):S14-80.

4. Lanza FL, Chan FK, Quigley EM, Practice Parameters Committee of the American College of G. Guidelines for prevention of NSAID-related ulcer complications. Am J Gastroenterol 2009;104:728-38.

5. Mukherjee D, Nissen SE, Topol EJ. Risk of cardiovascular events associated with selective COX-2 inhibitors. JAMA 2001;286:954-9.

6. De Berardis G, Lucisano G, D'Ettorre A, et al. Association of aspirin use with major bleeding in patients with and without diabetes. JAMA 2012;307:2286-94.

7. Sirois $\mathrm{C}$, Moisan J, Poirier $\mathrm{P}$, et al. Myocardial infarction and gastro-intestinal bleeding risks associated with aspirin use among elderly individuals with type 2 diabetes. Ann Med 2014;46:335-40.

8. Ray WA. Evaluating medication effects outside of clinical trials: new-user designs. Am J Epidemiol 2003;158:915-20.

9. Garcia Rodriguez LA, Hernandez-Diaz S. Relative risk of upper gastrointestinal complications among users of acetaminophen and nonsteroidal anti-inflammatory drugs. Epidemiology 2001;12:570-6.

10. Austin PC, Grootendorst P, Anderson GM. A comparison of the ability of different propensity score models to balance measured variables between treated and untreated subjects: a Monte Carlo study. Stat Med 2007;26:734-53.

11. Schneeweiss S, Rassen JA, Glynn RJ, et al. High-dimensional propensity score adjustment in studies of treatment effects using health care claims data. Epidemiology 2009;20:512-22.

12. Garbe $E$, Kloss $S$, Suling $M$, et al. High-dimensional versus conventional propensity scores in a comparative effectiveness study of coxibs and reduced upper gastrointestinal complications. Eur $J$ Clin Pharmacol 2013;69:549-57.

13. Austin PC. Balance diagnostics for comparing the distribution of baseline covariates between treatment groups in propensity-score matched samples. Stat Med 2009;28:3083-107.

14. Fine JP, Gray RJ. A proportional hazards model for the subdistribution of a competing risk. J Am Stat Assoc 1999:94:496-509.

15. Targownik LE, Metge CJ, Leung S, et al. The relative efficacies of gastroprotective strategies in chronic users of nonsteroidal anti-inflammatory drugs. Gastroenterology 2008;134:937-44.

16. Wu CY, Wu MS, Kuo KN, et al. Long-term peptic ulcer rebleeding risk estimation in patients undergoing haemodialysis: a 10-year nationwide cohort study. Gut 2011;60:1038-42.

17. Fiaccadori E, Maggiore U, Clima B, et al. Incidence, risk factors, and prognosis of gastrointestinal hemorrhage complicating acute renal failure. Kidney Int 2001;59:1510-19.

18. Fujita $\mathrm{T}$, Kutsumi $\mathrm{H}$, Sanuki $\mathrm{T}$, et al. Adherence to the preventive strategies for nonsteroidal anti-inflammatory drug- or low-dose aspirin-induced gastrointestinal injuries. $J$ Gastroenterol 2013:48:559-73.
19. Chan FK, Lanas A, Scheiman J, et al. Celecoxib versus omeprazole and diclofenac in patients with osteoarthritis and rheumatoid arthritis (CONDOR): a randomised trial. Lancet 2010;376:173-9.

20. Calvin AD, Aggarwal NR, Murad MH, et al. Aspirin for the primary prevention of cardiovascular events: a systematic review and meta-analysis comparing patients with and without diabetes. Diabetes Care 2009;32:2300-6.

21. Rocca B, Santilli F, Pitocco D, et al. The recovery of platelet cyclooxygenase activity explains interindividual variability in responsiveness to low-dose aspirin in patients with and without diabetes. J Thromb Haemost 2012;10:1220-30.

22. Linden MD, Tran HA. Overcoming aspirin treatment failure in diabetes. Crit Rev Clin Lab Sci 2012;49:183-98.

23. Chittaranjan A, Chethan KB, Sandarsh S. Cardiovascular mechanisms of SSRI drugs and their benefits and risks in ischemic heart disease and heart failure. Int Clin Psychopharmacol 2013;28:145-55.

24. Kimmel SE, Schelleman H, Berlin JA, et al. The effect of selective serotonin re-uptake inhibitors on the risk of myocardial infarction in a cohort of patients with depression. Br J Clin Pharmacol 2011;72:514-17.

25. Freedland KE, Carney RM. Depression as a risk factor for adverse outcomes in coronary heart disease. BMC Med 2013;11:131.

26. Kikuchi $\mathrm{Y}$, Iwase M, Fujii $\mathrm{H}$, et al. Association of severe hypoglycemia with depressive symptoms in patients with type 2 diabetes: the Fukuoka Diabetes Registry. BMJ Open Diabetes Res Care 2015;3: 000063 .

27. Shin $\mathrm{D}, \mathrm{Oh} \mathrm{YH}$, Eom $\mathrm{CS}$, et al. Use of selective serotonin reuptake inhibitors and risk of stroke: a systematic review and meta-analysis. $J$ Neurol 2014;261:686-95.

28. Scuteri A, Modestino A, Fedullo F, et al. Depression treatment selectively modifies arterial stiffness in older participants. $J$ Gerontol A Biol Sci Med Sci 2013;68:719-25.

29. Kemp AH, Brunoni AR, Santos IS, et al. Effects of depression, anxiety, comorbidity, and antidepressants on resting-state heart rate and its variability: an ELSA-Brasil cohort baseline study. Am J Psychiatry 2014; 171:1328-34.

30. Castellsague J, Pisa F, Rosolen V, et al. Risk of upper gastrointestinal complications in a cohort of users of nimesulide and other nonsteroidal anti-inflammatory drugs in Friuli Venezia Giulia, Italy. Pharmacoepidemiol Drug Saf 2013;22:365-75.

31. Castellsague J, Riera-Guardia N, Calingaert B, et al. Individual NSAIDs and upper gastrointestinal complications: a systematic review and meta-analysis of observational studies (the SOS project). Drug Saf 2012;35:1127-46.

32. Lee SH, Han CD, Yang IH, et al. Prescription pattern of NSAIDs and the prevalence of NSAID-induced gastrointestinal risk factors of orthopaedic patients in clinical practice in Korea. J Korean Med Sci 2011;26:561-7.

33. Shau WY, Chen HC, Chen ST, et al. Risk of new acute myocardial infarction hospitalization associated with use of oral and parentera non-steroidal anti-inflammation drugs (NSAIDs): a case-crossover study of Taiwan's National Health Insurance claims database and review of current evidence. BMC Cardiovasc Disord 2012;12:4.

34. Chang $\mathrm{CH}$, Shau WY, Kuo CW, et al. Increased risk of stroke associated with nonsteroidal anti-inflammatory drugs: a nationwide case-crossover study. Stroke 2010;41:1884-90.

35. Laporte JR, Ibanez L, Vidal X, et al. Upper gastrointestinal bleeding associated with the use of NSAIDs: newer versus older agents. Drug Saf 2004:27:411-20.

36. Spies CM, Stemmler E, Buttgereit F. How to mechanistically explain the CONDOR study data. Med Hypotheses 2015;84:14-19.

37. Gryglewski RJ, Dembinska-Kiec A, Korbut R. A possible role of thromboxane A2 (TXA2) and prostacyclin (PGI2) in circulation. Acta Biol Med Ger 1978;37:715-23.

38. Schneeweiss S, Glynn RJ, Tsai EH, et al. Adjusting for unmeasured confounders in pharmacoepidemiologic claims data using external information: the example of COX2 inhibitors and myocardial infarction. Epidemiology 2005;16:17-24

39. Crooks CJ, West J, Card TR. Comorbidities affect risk of nonvariceal upper gastrointestinal bleeding. Gastroenterology 2013;144:1384-93, 93 e1-2; quiz e18-9. 